\title{
Successful Treatment of Hepatocellular Carcinoma with Lung Metastasis Using Hepatic and Bronchial Artery Infusion Chemotherapy
}

\author{
Tatsuki Yoshida ${ }^{1}$, Kentaro Kamada ${ }^{1}$, Kouichi Miura ${ }^{1}$, Takashi Goto ${ }^{1}$, Shigetoshi Ohshima ${ }^{1}$, \\ Wataru Sato ${ }^{1}$, Tomomi Shibuya ${ }^{1}$, Takahiro Dohmen ${ }^{1}$, Ryo Kanata ${ }^{1}$, Toshitaka Sakai ${ }^{1}$, \\ Mitsuru Chiba ${ }^{1}$, Junichi Fujiwara ${ }^{1}$, Yuko Sugimoto ${ }^{1}$, Mitsuaki Ishioka ${ }^{1}$, \\ Itsuki Hasegawa ${ }^{1}$, Kenichi Takahashi ${ }^{1}$, Shinichiro Minami ${ }^{1}$, \\ Akashi Fujita ${ }^{1}$, Manabu Hashimoto ${ }^{2}$ and Hirohide Ohnishi ${ }^{1}$
}

\begin{abstract}
We herein report a case of hepatocellular carcinoma (HCC) with lung metastasis that was successfully treated with transcatheter arterial infusion chemotherapy via the hepatic and bronchial arteries. A 64-year-old man diagnosed with HCC in 2003 was treated with locoregional therapy followed by sorafenib for recurrent HCC. Tumor thrombosis and lung metastasis were noted in April 2012. We administered IA-call ${ }^{\circledR}$, a finepowder formulation of cisplatin, via the hepatic and bronchial arteries. This therapy resulted in the disappearance of the lung metastases and a partial response to tumor thrombosis. The patient remained alive for 23 months after developing lung metastasis.
\end{abstract}

Key words: hepatocellular carcinoma, lung metastasis, hepatic arterial infusion chemotherapy, bronchial arterial infusion chemotherapy, second-line chemotherapy

(Intern Med 53: 2493-2497, 2014)

(DOI: 10.2169/internalmedicine.53.2957)

\section{Introduction}

Hepatocellular carcinoma ( $\mathrm{HCC}$ ) is a common cancer in Asia and Africa whose prevalence is also increasing in Western countries (1). There are several different treatment modalities for HCC, including transcatheter arterial chemoembolization (TACE), radiofrequency ablation (RFA), percutaneous ethanol injection (PEI) and partial hepatectomy for HCC localized to the liver. These treatments improve the survival of HCC patients by controlling locally developed HCC lesions (2-5). On the other hand, clinicians frequently encounter HCC patients with extrahepatic metastasis as a result of prolonged survival. The prognosis of HCC patients with extrahepatic metastasis is poor. Indeed, the median survival time and 1-year survival rate of such patients are only
4.9-7 months and $21.7-24.9 \%$, respectively $(6,7)$. According to the treatment algorithm provided by the Japanese Society of Hepatology (JSH) (8) and the American Association for the Study of Liver Disease (AASLD) (9), sorafenib is the only choice for HCC with extrahepatic lesions in cases in which the background liver cirrhosis is Child-Pugh grade A. However, the rate of response to sorafenib, including a complete response (CR) and partial response (PR), is $8 \%$, as assessed according to the modified Response Evaluation Criteria in Solid Tumors, while the median time to radiological progression is 4.2 months in HCC patients with extrahepatic metastasis (10). Although the administration of second-line treatment following sorafenib is necessary, such regimens have not yet been established.

We herein report the successful treatment of HCC with lung metastasis using repeated transcatheter arterial infusion

${ }^{1}$ Department of Gastroenterology and Neurology, Akita University Graduate School of Medicine, Japan and ${ }^{2}$ Department of Radiology, Akita University Graduate School of Medicine, Japan

Received for publication March 26, 2014; Accepted for publication July 6, 2014

Correspondence to Dr. Kouichi Miura, miura116@doc.med.akita-u.ac.jp 

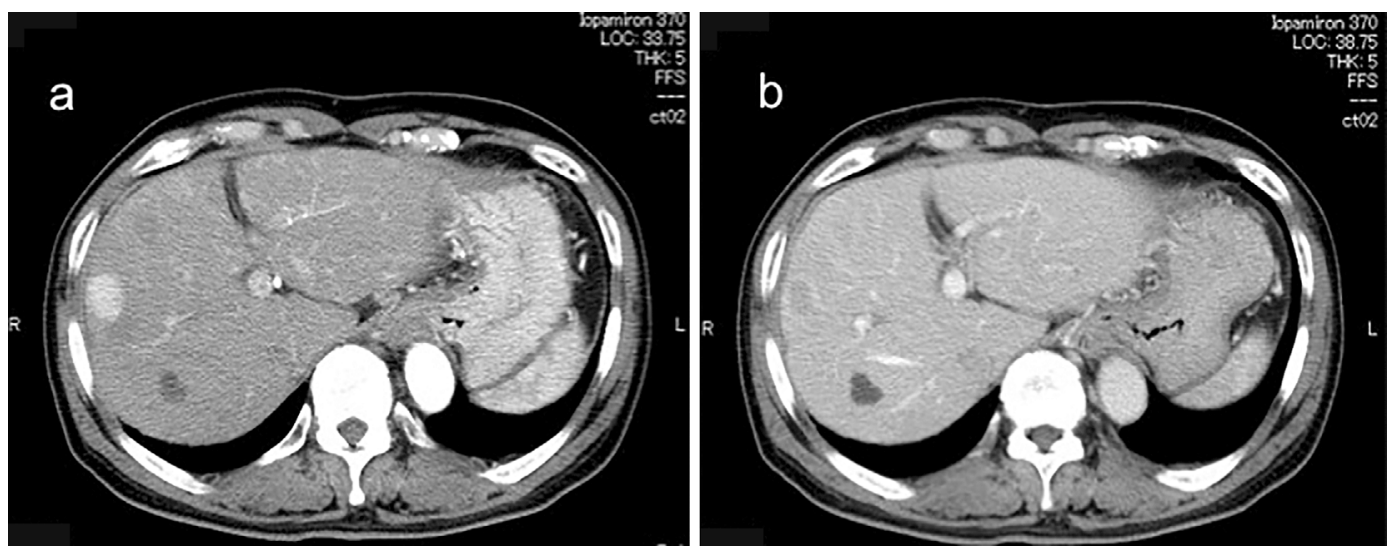

Figure 1. Contrast-enhanced CT scan demonstrates a hypervascular tumor in segment 8 in the arterial phase (a). Washout of contrast material is noted in the portal phase (b).
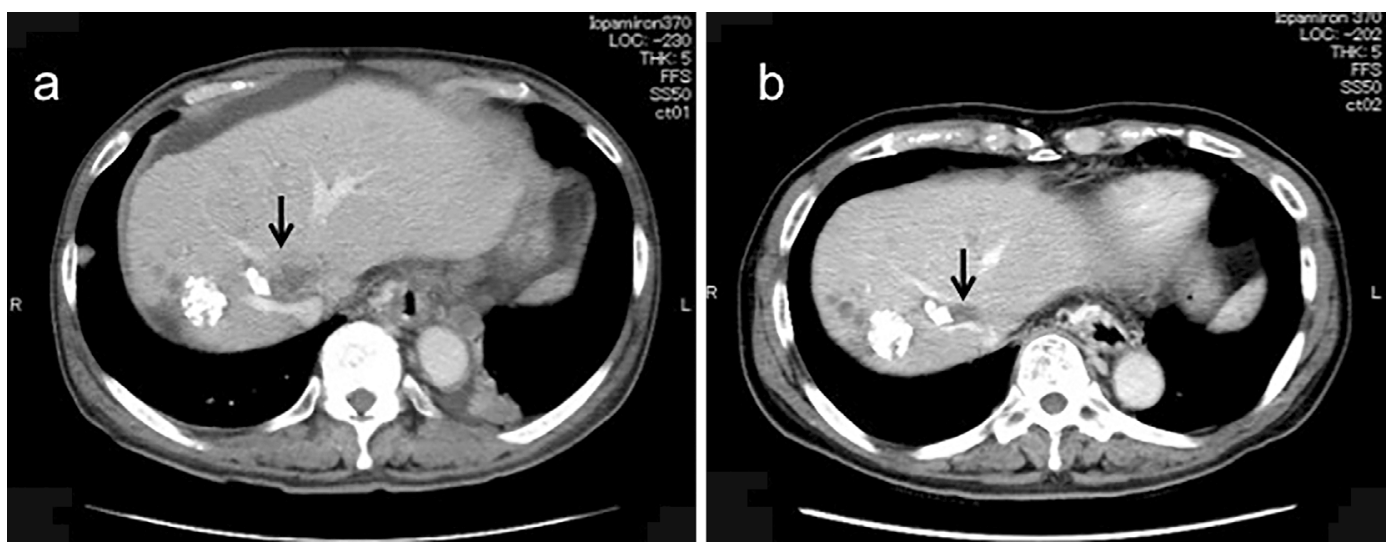

Figure 2. Portal phase of a contrast-enhanced CT scan displays HCC with venous invasion into the middle hepatic vein (a, arrow). The volume of the primary tumor and tumor thrombosis were reduced by TAI combined with BAI after eight courses of chemotherapy (b, arrow).

chemotherapy (TAI) combined with bronchial arterial infusion chemotherapy (BAI) in a patient who achieved progressive disease (PD) after receiving sorafenib.

\section{Case Report}

A 64-year-old man with hepatitis $\mathrm{C}$ virus-related liver cirrhosis was diagnosed as having a single nodular $\mathrm{HCC}$ in segment 5 in January 2003. The HCC lesion measured 24 $\mathrm{mm}$ in diameter, and the grade of liver damage was ChildPugh A. The patient was treated with TACE followed by PEI at a local hospital, as he rejected hepatectomy. Thereafter, he was treated with locoregional therapy, including TACE and RFA, for recurrent HCC in 2005 and 2006. These treatments resulted in the disappearance of HCC and provided an opportunity to administer pegylated-interferon plus ribavirin therapy; however, the interferon therapy did not eradicate the HCV. In January 2008, the patient was referred to Akita University Hospital for the treatment of recurrent HCC. Upon admission, a tumor measuring $20 \mathrm{~mm}$ in size was found to be located in segment 8 (Fig. 1). Although the background liver cirrhosis remained Child-Pugh grade $\mathrm{A}$, the patient was treated with TACE according to his request. Subsequently, locoregional therapy was administered for recurrent HCC in December 2008, 2009 and 2010. The TACE procedure included the use of epirubicin-lipiodol emulsion. In December 2010, treatment with sorafenib at a daily dose of $800 \mathrm{mg}$ was started, as the locoregional therapies were unable to overcome the growth of HCC. The sorafenib treatment achieved PR for six months with subsequent stable disease (SD) for three months. However, the levels of $\alpha$-fetoprotein (AFP) and protein induced by Vitamin $\mathrm{K}$ absence or antagonists-II (PIVKA-II) became elevated in October 2011, reaching 10,884 ng/mL and 1,892 $\mathrm{mAU} / \mathrm{mL}$, respectively, in April 2012. A CT scan showed tumor thrombosis in the middle hepatic vein (Fig. 2a). In addition, multiple lung metastases were detected in both lungs (Fig. 3a). Therefore, sorafenib was discontinued due to PD. However, we considered that additional treatment would prolong the patient's survival because the grade of liver damage had remained at Child-Pugh A. Under informed consent, a bolus injection of IA-call ${ }^{\circledR}$ (Nippon Kayaku, Tokyo, Japan), a fine-powder formulation of cisplatin, was administered via the hepatic and bronchial arteries in one session: up to $25 \mathrm{mg}$ of IA-call ${ }^{\circledR}$ was injected via the right hepatic artery over three to 10 minutes, left hepatic ar- 

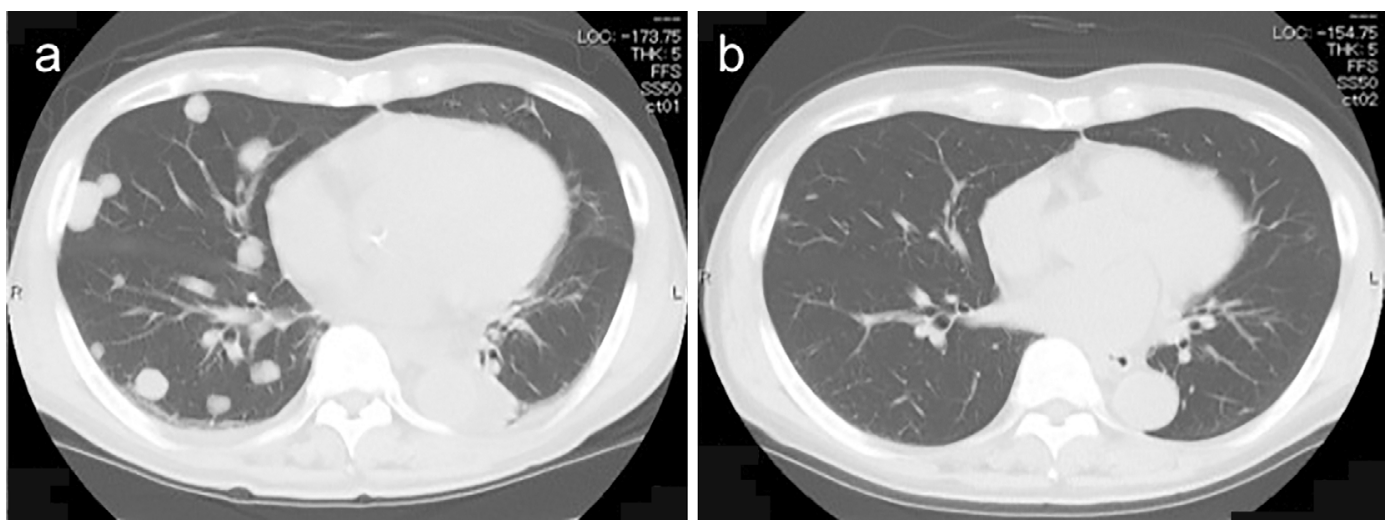

Figure 3. Chest CT shows multiple metastatic lesions of $\mathrm{HCC}$ in the lungs (a). No nodules were found after eight courses of TAI combined with BAI (b).

TAl+BAI
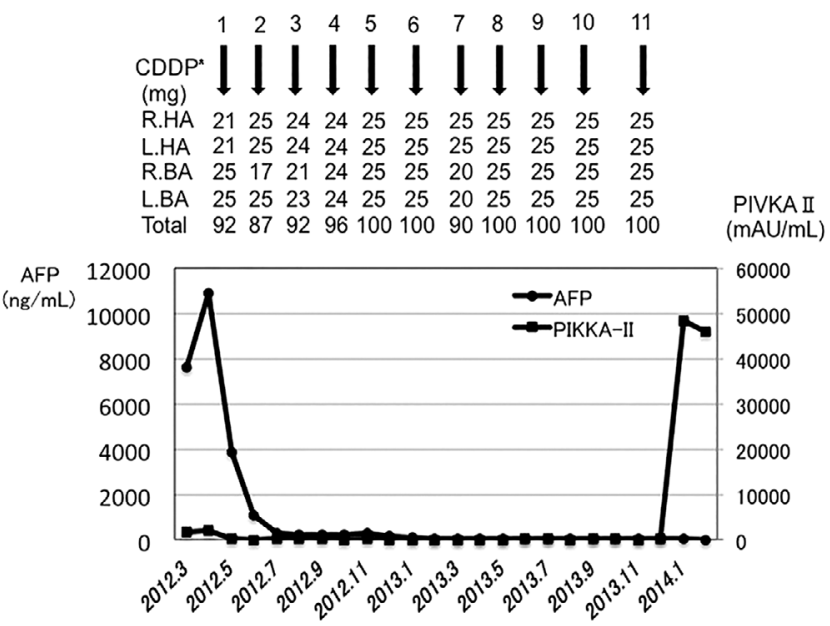

Figure 4. Clinical course of the patient after resistance to sorafenib. The upper panel shows the timing and dosage of cisplatin ( $\mathrm{mg}$ ) in the arterial infusion chemotherapy regimen. The lower panel shows the levels of tumor markers, including AFP and PIVKA-II. The tumor markers promptly decreased in response to the initial treatment with TAI combined with BAI and remained near the normal range following three courses of chemotherapy. However, the PIVKA-II level was found to be sharply elevated in January 2014, soon before the patient's death. CDDP*: cisplatin (IA-call ${ }^{\circledR}$ ), R.HA: right hepatic artery, L.HA: left hepatic artery, R.BA: right bronchial artery, L.BA: left bronchial artery

tery over three to 10 minutes, right bronchial artery over six to 10 minutes and left bronchial artery over six to $10 \mathrm{~min}$ utes, respectively. No respiratory symptoms were noted following this combination treatment with BAI. The initial arterial infusion chemotherapy promptly lowered the AFP and PIVKA-II levels to $6,162.9 \mathrm{ng} / \mathrm{mL}$ and $178 \mathrm{mAU} / \mathrm{mL}$, respectively. Therefore, therapy with IA-call ${ }^{\circledR}$, up to $100 \mathrm{mg}$ per session, was continued every six to eight weeks with monitoring of the bone marrow and renal functions. After two courses of the combined chemotherapy, the AFP and
PIVKA-II levels dropped to almost the normal range, then maintained within the normal limits (Fig. 4). In addition, the number and size of lung metastases dramatically decreased, and the metastatic lesions disappeared after eight courses of therapy (Fig. 3b). In contrast, although the tumor thrombosis decreased in volume, it did not disappear (Fig. 2b). Therefore, we continued the chemotherapy regimen in order to prevent regrowth of the primary tumors and the recurrence of metastasis. However, the interval to the 11th course of chemotherapy was extended due to a delayed recovery of the renal function. While awaiting the next course of chemotherapy, the patient developed refractory ascites, with sharp elevation of the PIVKA-II level in January 2014 (Fig. 4). A CT scan demonstrated no masses in the lungs or liver, with the exception of tumor thrombosis, suggesting that residual tumor cells had spread to the ascites and/or infiltrated diffusely into the liver. Ultimately, the patient died 23 months after developing tumor thrombosis and lung metastasis.

\section{Discussion}

We herein demonstrated the successful treatment of HCC associated with lung metastasis using TAI combined with BAI in a patient resistant to sorafenib. We selected this chemotherapy for the following reasons: first, additional treatment was expected to prolong the patient's survival (11) because the degree of liver damage remained Child-Pugh grade A; second, TAI often provides beneficial effects for HCC associated with tumor thrombosis (12); and third, the lung metastasis was likely to threaten the patient's life because the metastatic lesions were rapidly expanding. TAI is traditionally performed in Japan for advanced HCC, in particular that occurring with tumor thrombosis. A recent nationwide study in Japan demonstrated that TAI prolongs overall survival compared with non-active treatment in patients with advanced HCC (11). Ando et al. reported that the rate of response to TAI, including $\mathrm{CR}$ and $\mathrm{PR}$, is $48 \%$, with a 1-year survival rate of $45 \%$, in HCC patients with portal vein tumor thrombosis (12). Therefore, the JSH recommends 
Table. Successful Treatment Cases with BAI for Lung Metastasis of HCC

\begin{tabular}{|c|c|c|c|c|}
\hline Ref & Regimen of BAI & Session & Follow-up & Outcome \\
\hline (23) & CDDP 50mg, MMC 6mg & 2 & 16 months & Alive \\
\hline (24) & CDDP 50mg, MMC 10mg, ADR 20mg & 2 & 6 years & Alive \\
\hline$(25)$ & EPI $50 \mathrm{mg}$ & 1 & 14 months & Alive \\
\hline$(26)$ & CDDP 20mg, MMC 4mg, EPI 20mg & 2 & 30 months & Alive \\
\hline (27) & $\mathrm{MMC} 10 \mathrm{mg}$ & 3 & 61 months & Died \\
\hline (28) & EPI, then CDDP* 80mg & 6 & 6 years & Alive \\
\hline Present case & CDDP* 50mg & 11 & 23 months & Died \\
\hline
\end{tabular}

that TAI be used as a treatment option in patients with HCC when locoregional therapy is unable to control the HCC lesions. In addition, TAI has also beneficial effects against advanced HCC following sorafenib treatment $(13,14)$. The protocols for TAI are roughly divided into three regimens: $5 \mathrm{FU}$ plus low-dose cisplatin, 5FU plus interferon, and the single use of cisplatin (15). The effects of TAI are variable, with a response rate of $8-71 \%$ and median survival of 615.9 months (15). In the present case, we used a bolus injection of cisplatin for the primary HCC and lung metastasis in the TAI and BAI regimens, as described below. Although the rate of response to systemic cisplatin administration is only $9.3 \%$ (16), that for the cisplatin used in TAI regimens is much higher at $33.8 \%$, with a 1-year survival rate reaching $67.5 \%$ (17). However, the rate of response to cisplatin in TAI regimens decreases to only $3.6 \%$ when the enrolled patients are limited to those with TACE-refractory HCC (18).

Metastatic HCC can be life-threatening (6), although the stage of intrahepatic HCC is a significant and independent prognostic factor (10). Therefore, we added BAI to the treatment regimen for lung metastasis in the present case because BAI has the advantage of delivering a high concentration of drugs to the bronchial arteries, which supply arterial blood to metastatic lesions. BAI was developed as a therapeutic modality for unresectable primary lung cancer and was frequently used in cases of primary lung cancer in the 1960 's $(19,20)$. However, the use of BAI is currently limited because its effects are not always superior to those of other treatment modalities. Therefore, the current treatment algorithm for primary lung cancer does not include BAI. On the other hand, BAI is often used to treat metastatic lung cancer, with dramatic effects $(21,22)$. Table provides a summary of successful cases, including the present case, of treatment with BAI for lung metastasis of HCC (23-28). Five patients were treated with BAI only because the primary HCCs were well controlled. Kogure et al. reported that TAI combined with simultaneous BAI can achieve CR, even in cases involving primary HCC lesions (26). All seven cases exhibited beneficial outcomes without adverse respiratory effects following therapy with cisplatin at a dose up to $80 \mathrm{mg}$ in BAI, whereas a higher dose often results in the formation of bronchial ulcers (29). Although sufficient evidence is not yet available regarding the effects of BAI for pulmonary metastasis of HCC, this treatment is nevertheless considered to be a potentially effective modality in such cases.

Second-line chemotherapy regimens intended for use after sorafenib have not yet been established. Although sorafenib may prolong the survival of patients with advanced HCC, the response rate is extremely low (30). New moleculartargeted drugs remain under investigation, including everolimus, brivanib, ramucirumab, axitinib and tivantinib (15). Of these agents, tivantinib, a c-MET inhibitor, is a candidate for application in second-line chemotherapy regimens following sorafenib (31). Because these drugs are not commercially available at present, we administered cisplatin as the second-line regimen in this case using the arterial infusion technique. There are major differences from the standpoint of arterial infusion chemotherapy between Western countries and Japan. According to the guidelines of the AASLD, TAI is not recommended as either first- or second-line chemotherapy. This is because the TAI regimen was developed based on empirical evidence. In contrast, the guidelines established by the JSH allow for the administration of TAI as a first-line chemotherapy if locoregional therapies fail to control intrahepatic HCC lesions.

In summary, we herein presented a case of successful treatment of advanced HCC with lung metastasis using TAI combined with BAI. This treatment modality has the potential to be used as a second-line chemotherapy for advanced HCC associated with lung metastasis following sorafenib treatment. However, further studies are required to determine the efficacy of TAI combined with BAI for treating HCC with lung metastasis.

The authors state that they have no Conflict of Interest (COI).

\section{References}

1. Kim DY, Han KH. Epidemiology and surveillance of hepatocellular carcinoma. Liver Cancer 1: 2-14, 2012.

2. Huang GT, Lee PH, Tsang YM, et al. Percutaneous ethanol injection versus surgical resection for the treatment of small hepatocellular carcinoma: a prospective study. Ann Surg 242: 36-42, 2005.

3. Chen MS, Li JQ, Zheng Y, et al. A prospective randomized trial comparing percutaneous local ablative therapy and partial hepatectomy for small hepatocellular carcinoma. Ann Surg 243: 321-328, 2006.

4. Hasegawa K, Kokudo N, Makuuchi M, et al. Comparison of resection and ablation for hepatocellular carcinoma: a cohort study based on a Japanese nationwide survey. J Hepatol 58: 724-729, 
2013.

5. Takayasu K, Arii S, Ikai I, et al. Prospective cohort study of transarterial chemoembolization for unresectable hepatocellular carcinoma in 8510 patients. Gastroenterology 131: 461-469, 2006.

6. Uka K, Aikata H, Takaki S, et al. Clinical features and prognosis of patients with extrahepatic metastases from hepatocellular carcinoma. World J Gastroenterol 21: 414-420, 2007.

7. Natsuizaka M, Omura T, Akaike T, et al. Clinical features of hepatocellular carcinoma with extrahepatic metastases. J Gastroenterol Hepatol 20: 1781-1787, 2005.

8. Kudo M, Izumi N, Kokudo N, et al. Management of hepatocellular carcinoma in Japan: Consensus-Based Clinical Practice Guidelines proposed by the Japan Society of Hepatology (JSH) 2010 updated version. Dig Dis 29: 339-364, 2011.

9. Bruix J, Sherman M; American Association for the Study of Liver Diseases. Management of hepatocellular carcinoma: an update. Hepatology 53: 1020-1022, 2011.

10. Kawaoka T, Aikata H, Kan H, et al. Clinical outcome and prognostic factors of patients with hepatocellular carcinoma and extrahepatic metastasis treated with sorafenib. Hepatol Res (in press).

11. Nouso K, Miyahara K, Uchida D, et al. Effect of hepatic arterial infusion chemotherapy of 5-fluorouracil and cisplatin for advanced hepatocellular carcinoma in the Nationwide Survey of Primary Liver Cancer in Japan. Br J Cancer 109: 1904-1907, 2013.

12. Ando E, Tanaka M, Yamashita F, et al. Hepatic arterial infusion chemotherapy for advanced hepatocellular carcinoma with portal vein tumor thrombosis: analysis of 48 cases. Cancer 95: 588-595, 2002.

13. Terashima T, Yamashita T, Arai K, et al. Feasibility and efficacy of hepatic arterial infusion chemotherapy for advanced hepatocellular carcinoma after sorafenib. Hepatol Res (in press).

14. Jeong SW, Jang JY, Lee JE, et al. The efficacy of hepatic arterial infusion chemotherapy as an alternative to sorafenib in advanced hepatocellular carcinoma. Asia Pac J Clin Oncol 8: 164-171, 2012.

15. Kudo M. Treatment of advanced hepatocellular carcinoma with emphasis on hepatic arterial infusion chemotherapy and molecular targeted therapy. Liver Cancer 1: 62-70, 2012.

16. Nagahama H, Okada S, Okusaka T, et al. Predictive factors for tumor response to systemic chemotherapy in patients with hepatocellular carcinoma. Jpn J Clin Oncol 27: 321-324, 1997.

17. Yoshikawa M, Ono N, Yodono H, Ichida T, Nakamura H. Phase II study of hepatic arterial infusion of a fine-powder formulation of cisplatin for advanced hepatocellular carcinoma. Hepatol Res 38: 474-483, 2008.

18. Iwasa $S$, Ikeda $M$, Okusaka $T$, et al. Transcatheter arterial infusion chemotherapy with a fine-powder formulation of cisplatin for advanced hepatocellular carcinoma refractory to transcatheter arterial chemoembolization. Jpn J Clin Oncol 41: 770-775, 2011.

19. Soderberg CH Jr, Colbert MP, Leone LA. Bronchial artery infu- sion therapy of lung neoplasms with nitrogen mustard. Surgery 56: 897-904, 1964.

20. Neyazaki T, Ikeda M, Seki Y, Egawa N, Suzuki C. Bronchial artery infusion therapy for lung cancer. Cancer 24: 912-922, 1969.

21. Koshiishi H, Yoshimura T, Okamura T, et al. Evaluation of bronchial arterial infusion (BAI) for metastatic lung tumor from colorectal cancer. Gan To Kagaku Ryoho (Jpn J Cancer Chemother) 31: 1838-1841, 2004 (in Japanese, Abstract in English).

22. Yonezawa M, Seki A, Numoto A, Kawada K, Eguchi K, Kudo T. Successful treatment of metastatic pulmonary tumors by bronchial arterial infusion chemotherapy in two patients with locally well controlled uterine cancer. Acta Med Okayama 48: 109-112, 1994.

23. Komada H, Yamamura M, Kawaguchi $Y$, et al. Disappearance of lung metastases from hepatocellular carcinoma following bronchial arterial infusion of CDDP and MMC. Gan To Kagaku Ryoho (Jpn J Cancer Chemother) 19 (10 Suppl): 1504-1507, 1992 (in Japanese, Abstract in English).

24. Kawasaki H, Araki K, Hamada S, et al. A case of long survival of hepatocellular carcinoma with lung metastases after right trisegmentectomy due to multi-disciplinary treatment. Shoukaki Geka (Gastroenterological Surgery) 16: 1603-1610, 1993 (in Japanese).

25. Uetsuji S, Yamada O, Komada H, et al. Disappearance of lung metastases from hepatocellular carcinoma after bronchial arterial infusion of epirubicin. Gan No Rinshou (Jpn J Cancer Clin) 42: 181-184, 1996 (in Japanese, Abstract in English).

26. Kogure T, Iwasaki T, Ueno Y, et al. Complete remission of a case of hepatocellular carcinoma with tumor invasion in inferior vena cava and with pulmonary metastasis successfully treated with repeated arterial infusion chemotherapy. Hepatogastroenterology 54: 2113-2116, 2007.

27. Sakamoto H, Amikura K, Tanaka Y, et al. Successful treatment of multiple pulmonary and peritoneal recurrence of hepatocellular carcinoma with bronchial artery infusion therapy and PEIT followed by surgery: a case report. Gan To Kagaku Ryoho (Jpn J Cancer Chemother) 37: 2687-2689, 2010 (in Japanese, Abstract in English).

28. Koyama J, Morizono R, Goto T, et al. A therapeutic effective case of bronchial arterial infusion of fine-powder cisplatin (IA-call) for pulmonary metastases originated from hepatocellular carcinoma. Kanzo (Liver) 54: 19-26, 2013 (in Japanese, Abstract in English).

29. Osaki T, Hanagiri T, Nakanishi R, Yoshino I, Taga S, Yasumoto K. Bronchial arterial infusion is an effective therapeutic modality for centrally located early-stage lung cancer: results of a pilot study. Chest 115: 1424-1428, 1999.

30. Llovet JM, Ricci S, Mazzaferro V, et al. Sorafenib in advanced hepatocellular carcinoma. N Engl J Med 359: 378-390, 2008.

31. Rimassa L, Personeni N, Simonelli M, Santoro A. Tivantinib: a new promising mesenchymal-epithelial transition factor inhibitor in the treatment of hepatocellular carcinoma. Future Oncol 9: 153$165,2013$.

(C) 2014 The Japanese Society of Internal Medicine http://www.naika.or.jp/imonline/index.html 